\title{
Bit Allocation for Lossy Image Set Compression
}

\author{
Howard Cheng \\ Department of Mathematics and Computer Science \\ University of Lethbridge \\ Lethbridge, Alberta, T1K 3M4, Canada \\ Email: howard.cheng@uleth.ca
}

\author{
Camara Lerner \\ Department of Mathematics and Computer Science \\ University of Lethbridge \\ Lethbridge, Alberta, T1K 3M4, Canada \\ Email: camara.lerner@uleth.ca
}

\begin{abstract}
Large sets of similar images are produced in many applications. To store these images more efficiently, redundancy among similar images need to be exploited. A number of methods have been proposed to reduce such inter-image redundancy in lossy image set compression. These methods encode each image either using a conventional image compression algorithm, or predicts the image from a similar image already encoded and encode the prediction residual. Although these methods differ in the way they determine the prediction structure in the image set, they do not consider the effect of bit allocation on the overall quality of the reconstructed images. In this paper, we show that Lagrangian optimization can be used to determine bit allocation for each encoded image in order to improve the overall quality of the reconstructed image set. Furthermore, a model approximating rate-distortion curves of the residual images can be used to reduce the encoding time significantly.
\end{abstract}

\section{INTRODUCTION}

Large sets of similar images occur in many practical applications, including medical image databases, satellite remote sensing images, and photo albums [1]-[15]. Instead of compressing each image independently using conventional image compression algorithms, inter-image redundancy can be exploited to compress sets of similar images more efficiently.

A number of different approaches have been proposed to compress sets of similar images [1]-[10], [13]-[15]. In most of these works, the focus is on reducing inter-image redundancy by some variation of predictive coding - in order to compress an image $I$ in the set, a particular reference image $r(I)$ already encoded is used to predict $I$ so that only the prediction error (residual) image needs to be compressed. The residual image is compressed with conventional image or video compression algorithms. The main difference among these image set compression algorithms is the way the reference image $r(I)$ is chosen for each image $I$ in the set. We call this the prediction structure of the image set compression algorithm. Some of these methods have a fixed prediction structure that works well on some image sets but not others, while some have structures (often based on graphs) that adapt to a particular image set at the expense of more computation.

Most of these algorithms do not explicitly take bit allocation into account. That is, in order to achieve an overall target bit rate for the entire image set, the same bit rate is used to compress each residual image. Methods that use video compression algorithms to encode residual images may implicitly consider

978-1-4673-7788-1/15/\$31.00 (C)2015 IEEE bit allocation because such optimization is performed in some video compression algorithms, but no explicit consideration on bit allocation is given. As a result, the overall distortion in the reconstructed images may be higher than necessary for a particular bit rate.

In this paper, we consider the bit allocation problem explicitly in image set compression. Intuitively, if a residual image is easy to compress (e.g. all zero), we do not need to allocate many bits to it. The extra bits freed up in the overall bit budget can be used to encode other residual images that are more difficult to compress. Our objective is to achieve a target bit rate while minimizing overall distortion in the reconstructed images. Bit allocation strategies have been studied extensively in image and video compression [16]. For image set compression, we will study the bit allocation problem for the Centroid algorithm [4], [6], the MST and the $\mathrm{MST}_{a}$ algorithm [17]-[19]. By studying the rate-distortion curves of the residual images, we can use Lagrangian optimization to determine the bit allocation to compress each individual residual image. Some preliminary feasibility results have previously been shown [20] but the rate-distortion curves needed to be computed experimentally by sampling at many different bit rates for each residual image. This requires many invocations of the underlying image compression and decompression algorithms, and can be computationally very intensive. We show in this paper that the rate-distortion curves can be approximated in order to reduce the encoding time significantly. When wavelet packet compression [21] is used as the underlying image compression algorithm, we find that the approximations produce a very good result at a fraction of the computational time compared to our previous work [20].

To the best of our knowledge, the only other work that considered rate-distortion explicitly is a recent approach by Ling et al. [15], though they fixed a certain quantization level and assumed that the distortion is approximately the same for each residual image in order to simplify the optimization process. Their work only considered one quantization level for the image set, and do not consider the possibility of using different quantization levels for each residual image.

The paper is organized as follows. Some of the previous approaches studied in this paper are briefly reviewed in Section II. In Section III, our bit allocation problem is defined and our approach is described. The experimental results are shown in Section IV. 


\section{Image Set Compression Methods}

For sets of similar images, a prediction structure is used to determine which images are encoded directly and which images are predicted from an already encoded image, so that the prediction residual images are encoded instead. This is known as a set mapper [4], [6]. Both the original and residual images have to be encoded by an image compression algorithm. A conventional image compression algorithm such as JPEG2000 [22] is often used, although there are also some approaches that order these images into a sequence to be processed by a video compression algorithm (see for example, [15]). We briefly outline a few set mappers that have been proposed.

In the centroid method [4], [6], a centroid (average) image is computed and encoded directly. The remaining images are predicted from the centroid image, and the residual images are encoded. If all images are very similar, the residual images contain mostly zeroes and can be compressed very efficiently. The centroid method was designed for lossless compression but has been adapted to lossy compression [17]-[19].

Graph theoretical methods have also been proposed to compute the prediction structure. The vertices represent images and the edges give a "cost" of encoding the residual image of one image if it is predicted from the other image on the edge. For example, this cost may be the quality that can be achieved when the residual image is encoded at a particular bit rate, or vice versa. A minimum spanning tree is computed to represent the optimal prediction structure. This is called the MST method [17]-[19]. This method is useful when there is significant similarity between certain pairs of images in the image set, even if the images entire set is not necessarily very similar to each other. For certain image sets, it is also beneficial to add the centroid image before the minimum spanning tree is computed (called the $\mathrm{MST}_{a}$ method). Ling et al. [15] also used a graph theoretical approach, though the prediction structures of interest are depth-limited minimum spanning forests in order to limit the decompression time for any particular image in the set.

There are also set mappers designed for specific types of images. Some of these mappers use additional information to determine the prediction structure. They may also use more sophisticated prediction techniques based on the information. For example, for satellite images with large overlap of geographic areas, the geographical location of the image can be used to determine the prediction structure. In addition, adjustment for seasonal variations is needed to obtain effective prediction [10]. For multi-view images inter-view dependencies can be used to obtain better predictions [2], [23]. There are also special techniques for stereo images [24], [25].

\section{A. Distortion Measures}

In lossy image compression, the goal is to achieve the highest quality possible at a particular bit rate. The quality is generally measured by a distortion measure indicating how close the decompressed image is compared to the original image. One measure we examine in this work is the wellknown Root-Mean-Square-Error (RMSE) measure [26]. Although it does not always correspond to human perception, it is easy to compute and used by many researchers. Another measure that can be used, for example, is based on the Structural Similarity (SSIM) index [27]. It corresponds to human evaluation better, and a metric $\left(D_{2}\right)$ can be derived based on the SSIM index [28]. We will refer to this metric as the "SSIM measure" in this paper.

The MST-based algorithms also require edge weights in order to determine the prediction structure. As in the previous works [17], [19], we use the distortion between the two images as the edge weight - if two images are similar, then it should be easier to encode the corresponding prediction residual image. It has been shown that the MST-based algorithms are near-optimal over all algorithms based on pairwise prediction. This result is valid if the same distortion measure be used for both edge weights and quality evaluation. Thus, this will be the case for the remainder of this paper.

\section{Bit ALLOCATION}

We now formulate the optimization problem considered in lossy image set compression. We want to minimize the distortions between the decompressed images and the corresponding original images. In order to arrive at the optimal distortion, the bit allocation for encoding each residual image is varied in order to minimize the sum of the total distortions in the entire set. If a residual image is close to zero, it can be compressed at a lower bit rate without greatly affecting the distortion of that image. More bits can then be allocated to other residual images in order to minimize the sum of distortions.

Let $S=\left\{I_{1}, \ldots, I_{n}\right\}$ be a set of original or residual images chosen to be encoded by the prediction structure. We denote by $D(I, b)$ the distortion between $I$ and the decompressed image when $I$ is compressed using $b$ bits. The function $D(I, b)$ as $b$ varies is also called the rate-distortion curve of image $I$. If $B$ is the overall bit budget, the optimization problem of interest is:

$$
\begin{aligned}
& \text { minimize } \sum_{i=1}^{n} D\left(I_{i}, b_{i}\right), \\
& \text { subject to } \sum_{i=1}^{n} b_{i} \leq B,
\end{aligned}
$$

where $b_{i}$ is the number of bits used to encode image $I_{i}$. The distortion measure in our study is the RMSE or the SSIM measure (Section II). We call the solution to (1) the ratedistortion based bit allocation. It is common to assume that the compression of each residual is independent of each other in order to simplify the computation needed to find a solution to (1). However, this assumption is false in our setting as each image is predicted based on a previously encoded image.

There are a number of methods to obtain the solution of the optimization problem (1) [16]. In our work, we choose the commonly used method of Lagrangian optimization [29]. 
For each residual image, we minimize

$$
D\left(I_{i}, b_{i}\right)+\lambda b_{i},
$$

for some $\lambda \geq 0$. The point on the rate-distortion curve $D\left(I_{i}, b_{i}\right)$ with slope $-\lambda$ gives the bit rate $b_{i}$ minimizing (2). For a fixed $\lambda$, this gives the optimal bit allocation for the overall bit budget $B=\sum_{i=1}^{n} b_{i}$. Since the overall bit budget is not known a priori, one typically use binary search on $\lambda$ to solve (1) for a specific value of $B$.

In order to perform the Lagrangian optimization (2), the rate-disortion curve $D\left(I_{i}, b_{i}\right)$ must be known for each residual image. A simple approach is to simply invoke the chosen image compression algorithm on each residual image at various bit rates and record the distortions. In order to accurately solve the optimization problem (1), a large number of points on the rate-distortion curve are required. For example, each rate-distortion curve used in [20] were obtained using 15 different bit rates. This is computationally very intensive and impractical, especially if the underlying image compression algorithm is computationally intensive. This can be sped up by invoking the image compression algorithm on downscaled residual images [15] but that approach fixes a quantization setting and obtains only one point on the rate-distortion curve.

Mallat and Falzon [30] noted that for transform coding at low bit rate $(b<1 \mathrm{bit} / \mathrm{pixel})$, the rate-distortion varies like $b^{1-2 \gamma}$. Following this model, we approximate the ratedistortion curve of each residual image as

$$
D\left(I_{i}, b_{i}\right)=c_{i} b_{i}^{e_{i}}
$$

for some constants $c_{i}$ and $e_{i}$. In order to determine the constants for each residual image, the image compression algorithm is invoked on the residual image at a few bit rates. The distortion obtained at these bit rates are used to compute these constants with linear regression, by taking logarithms of (3):

$$
\log D\left(I_{i}, b_{i}\right)=e_{i} \log b_{i}+\log c_{i} .
$$

The number of invocations of the image compression and decompression algorithm can be reduced signifcantly by using this model. An additional benefit is that there is no need to use binary search to find the point on $D\left(I_{i}, b_{i}\right)$ with slope $-\lambda$ during the Lagrangian optimization process. The point $b_{i}$ can be determined analytically as

$$
b_{i}=\left(\frac{-\lambda}{c_{i} e_{i}}\right)^{\frac{1}{e_{i}-1}} .
$$

\section{EXPERIMENTAL RESUlts}

\section{A. Experiment Setup}

We performed our experiments using the centroid method [4], [6], the MST and $\mathrm{MST}_{a}$ methods [17]-[19]. In previous works, these methods have been shown to perform better than the "traditional" method of simply compressing each image independently of each other, even when each residual image is compressed at the same bit rate. The image compression algorithms used in this study are JPEG2000 [22] and wavelet packet compression [21]. We perform the experiments with both RMSE and the $D_{2}$ SSIM measure as the distortion measure.

Our approach is tested on four image sets that have been used in previous works [8], [9], [17], [19]. Figure 1 shows a typical image from the four image sets. The Joe set is a webcam image set taken from a camera directed at a beach in Victoria, British Columbia [31]. The Pig set is composed of ultrasound images of pig rib cages. The Galway set contains webcam images from a street in Galway City, Ireland [32]. Satellite images from the GOES project [33] make up the GOES set.
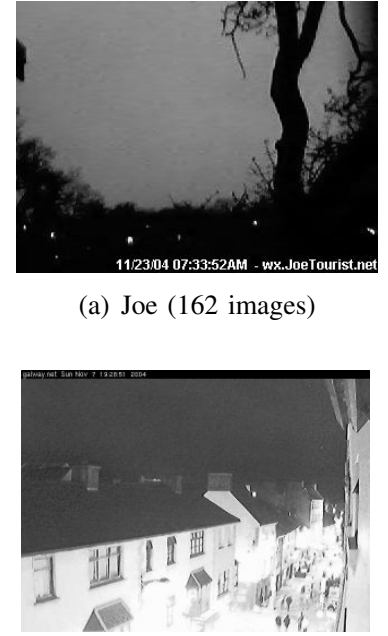

(c) Galway (28 images) (a) Joe (162 images)

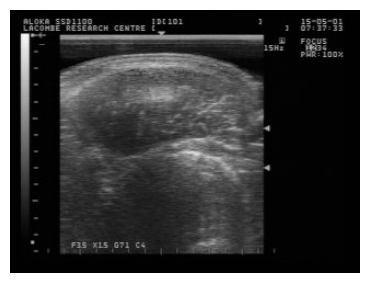

(b) Pig (304 images)

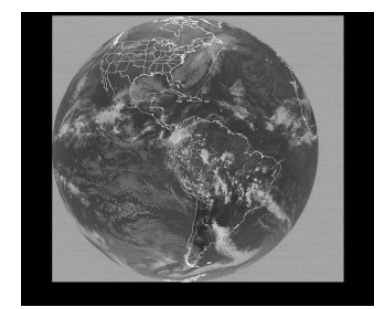

(d) GOES (128 images)
Fig. 1. Typical images from each set.

\section{B. Approximation of Rate-Distortion Curves}

We first present some evidence on the accuracy of the approximation (3) to the rate distortion curves. We invoked the compression algorithm at only 4 bit rates from $0.08 \mathrm{bpp}$ to $0.96 \mathrm{bpp}$ and obtained the model using linear regression. The model was then compared to distortion obtained at the 15 bit rates from $0.08,0.12,0.16, \ldots, 0.96 \mathrm{bpp}$ [20] to compute the coefficient of determination $r^{2}$. For brevity only the results for the Centroid method and the MST method using the RMSE distortion measure are shown in Table I, but the other methods gave similar results. It can be seen that the minimum $r^{2}$ values obtained are remarkably close to 1 , indicating that the approximations were very close to the acutal ratedistortion curves. Thus, using (3) as an approximation to the rate-distortion curve is acceptable and provides a significant reduction in computational time.

\section{Image Set Compression Results}

The proposed method is tested on the four image sets. First, we summarize the improvement in overall distortion using rate-distortion based bit allocation over equal allocation, when the overall bit budget are the same in both cases (Tables II, III, IV, and V). The target bit rates tested were $0.08,0.12,0.16$, 
TABLE I

Minimum $r^{2}$ VALUE OF RATE-DISTORTION CURVES APPROXIMATION. THE RMSE DISTORTION MEASURE IS USED.

\begin{tabular}{|c|r|r|r|r|}
\hline \multirow{2}{*}{} & \multicolumn{2}{|c|}{ JPEG2000 } & \multicolumn{2}{c|}{ Wavelet Packet } \\
\cline { 2 - 5 } & Centroid & MST & Centroid & MST \\
\hline Joe & 0.971 & 0.927 & 0.958 & 0.973 \\
\hline Pig & 0.956 & 0.871 & 0.907 & 0.934 \\
\hline Galway & 0.992 & 0.971 & 0.993 & 0.992 \\
\hline GOES & 0.962 & 0.860 & 0.969 & 0.956 \\
\hline
\end{tabular}

TABLE II

IMPROVEMENT IN RMSE USING RATE-DISTORTION BASED BIT ALLOCATION OVER EQUAL ALLOCATION. JPEG2000 WAS USED TO COMPRESS RESIDUAL IMAGES.

\begin{tabular}{|c|l|r|r|r|}
\hline \multicolumn{2}{|c|}{} & Centroid & MST & MST $_{a}$ \\
\hline \multirow{2}{*}{ Joe } & avg (\%) & 1.45 & 1.90 & 1.87 \\
& best (\%) & 7.34 & 4.09 & 4.31 \\
\hline \multirow{2}{*}{ Pig } & avg (\%) & -0.37 & 0.50 & -0.89 \\
& best (\%) & 11.25 & 2.78 & 1.43 \\
\hline \multirow{2}{*}{ Galway } & avg (\%) & 2.46 & 1.14 & 0.34 \\
& best (\%) & 9.18 & 4.32 & 7.03 \\
\hline \multirow{2}{*}{ GOES } & avg (\%) & 0.83 & 2.41 & 2.66 \\
& best (\%) & 3.46 & 5.39 & 5.27 \\
\hline
\end{tabular}

$\ldots, 0.96 \mathrm{bpp}$, and the rate-distortion curve for each residual image is obtained by sampling at these bit rates. From these tables, we see that important improvements were achieved in many cases. Generally, there is less improvement in the Pig set than the other sets, because most of the images in the Pig set are very similar. As a result, the rate-distortion curves for the residual images are all very similar, and equal bit allocation already achieves good results. Also, the Centroid method often benefits more from our bit allocation approach, because the centroid image needs to be more accurately encoded than others. In most cases, the improvement is more significant at lower target bit rates $(<0.40 \mathrm{bpp})$, because the allocation of each bit will have a more significant in the overall distortion when fewer bits are available.

We now examine the results when (3) is used to approximate the rate-distortion curves. Since the approximation is obtained using 4 bit rates, the time to compute the rate-distortion curves is significantly reduced by a factor of about 4 . The compression results showing the improvements of this approach compared to equal allocation are shown in Tables VI, VII, VIII, and IX.

TABLE III

IMPROVEMENT IN SSIM USING RATE-DISTORTION BASED BIT ALLOCATION OVER EQUAL ALLOCATION. JPEG2000 WAS USED TO COMPRESS RESIDUAL IMAGES.

\begin{tabular}{|c|l|r|r|r|}
\hline \multicolumn{2}{|c|}{} & Centroid & MST & MST $_{a}$ \\
\hline \multirow{2}{*}{ Joe } & avg (\%) & -0.97 & 0.59 & 0.53 \\
& best (\%) & 4.84 & 4.83 & 4.80 \\
\hline \multirow{2}{*}{ Pig } & avg (\%) & 0.73 & 1.18 & 1.04 \\
& best (\%) & 5.02 & 3.84 & 2.93 \\
\hline \multirow{2}{*}{ Galway } & avg (\%) & 1.86 & 0.75 & 0.83 \\
& best (\%) & 7.00 & 3.50 & 4.74 \\
\hline \multirow{2}{*}{ GOES } & avg (\%) & 0.22 & 1.75 & 1.73 \\
& best (\%) & 4.38 & 4.69 & 4.60 \\
\hline
\end{tabular}

TABLE IV

IMPROVEMENT IN RMSE USING RATE-DISTORTION BASED BIT ALLOCATION OVER EQUAL ALLOCATION. WAVELET PACKET COMPRESSION WAS USED TO COMPRESS RESIDUAL IMAGES.

\begin{tabular}{|c|l|r|r|r|}
\hline \multicolumn{2}{|c|}{} & Centroid & MST & MST $_{a}$ \\
\hline \multirow{2}{*}{ Joe } & avg (\%) & -0.79 & 0.45 & 0.23 \\
& best (\%) & 3.08 & 1.84 & 2.16 \\
\hline \multirow{2}{*}{ Pig } & avg (\%) & -1.69 & -0.31 & -1.72 \\
& best (\%) & 1.96 & 1.68 & 0.90 \\
\hline \multirow{2}{*}{ Galway } & avg (\%) & 1.97 & 2.03 & 2.51 \\
& best (\%) & 9.27 & 6.85 & 11.57 \\
\hline \multirow{2}{*}{ GOES } & avg (\%) & 2.26 & 4.28 & 4.98 \\
& best (\%) & 13.11 & 8.36 & 8.15 \\
\hline
\end{tabular}

TABLE V

IMPROVEMENT IN SSIM USING RATE-DISTORTION BASED BIT ALLOCATION OVER EQUAL ALLOCATION. WAVELET PACKET COMPRESSION WAS USED TO COMPRESS RESIDUAL IMAGES.

\begin{tabular}{|c|l|r|r|r|}
\hline \multicolumn{2}{|c|}{} & Centroid & MST & MST $_{a}$ \\
\hline \multirow{2}{*}{ Joe } & avg (\%) & -0.54 & -0.10 & -0.07 \\
& best (\%) & 3.67 & 1.92 & 1.97 \\
\hline \multirow{2}{*}{ Pig } & avg (\%) & -1.06 & -0.12 & -0.15 \\
& best (\%) & 1.72 & 0.62 & 1.24 \\
\hline \multirow{2}{*}{ Galway } & avg (\%) & 1.36 & 0.84 & 0.62 \\
& best (\%) & 6.66 & 3.89 & 6.16 \\
\hline \multirow{2}{*}{ GOES } & avg (\%) & 1.23 & 0.58 & 0.89 \\
& best (\%) & 11.91 & 2.33 & 2.10 \\
\hline
\end{tabular}

TABLE VI

IMPROVEMENT IN RMSE USING APPROXIMATED RATE-DISTORTION BASED BIT ALLOCATION OVER EQUAL ALLOCATION. JPEG2000 WAS USED TO COMPRESS RESIDUAL IMAGES.

\begin{tabular}{|c|l|r|r|r|}
\hline \multicolumn{2}{|c|}{} & Centroid & MST & MST $_{a}$ \\
\hline \multirow{2}{*}{ Joe } & avg (\%) & 1.93 & -0.16 & 0.13 \\
& best (\%) & 8.14 & 1.61 & 1.75 \\
\hline \multirow{2}{*}{ Pig } & avg (\%) & -3.53 & 0.23 & -1.72 \\
& best (\%) & -0.59 & 3.38 & 0.14 \\
\hline \multirow{2}{*}{ Galway } & avg (\%) & 0.84 & 0.01 & -1.09 \\
& best (\%) & 5.21 & 3.62 & 2.99 \\
\hline \multirow{2}{*}{ GOES } & avg (\%) & 1.28 & 0.46 & 0.96 \\
& best (\%) & 2.76 & 5.41 & 5.38 \\
\hline
\end{tabular}

TABLE VII

IMPROVEMENT IN SSIM USING APPROXIMATED RATE-DISTORTION BASED BIT ALLOCATION OVER EQUAL ALLOCATION. JPEG2000 WAS USED TO COMPRESS RESIDUAL IMAGES.

\begin{tabular}{|c|l|r|r|r|}
\hline \multicolumn{2}{|c|}{} & Centroid & MST & MST $_{a}$ \\
\hline \multirow{2}{*}{ Joe } & avg (\%) & 0.69 & -0.20 & -0.20 \\
& best (\%) & 4.64 & 0.97 & 1.03 \\
\hline \multirow{2}{*}{ Pig } & avg (\%) & -1.89 & -0.62 & -0.63 \\
& best (\%) & -0.41 & 0.92 & 0.70 \\
\hline \multirow{2}{*}{ Galway } & avg (\%) & 0.59 & -0.30 & -1.18 \\
& best (\%) & 4.11 & 0.45 & 0.15 \\
\hline \multirow{2}{*}{ GOES } & avg (\%) & -0.04 & -0.66 & -0.71 \\
& best (\%) & 3.51 & 1.13 & 1.10 \\
\hline
\end{tabular}


TABLE VIII

IMPROVEMENT IN RMSE USING APPROXIMATED RATE-DISTORTION BASED BIT ALLOCATION OVER EQUAL ALLOCATION. WAVELET PACKET COMPRESSION WAS USED TO COMPRESS RESIDUAL IMAGES.

\begin{tabular}{|c|l|r|r|r|}
\hline \multicolumn{2}{|c|}{} & Centroid & MST & MST $_{a}$ \\
\hline \multirow{2}{*}{ Joe } & avg (\%) & 0.90 & 0.54 & 0.47 \\
& best (\%) & 3.25 & 2.29 & 2.20 \\
\hline \multirow{2}{*}{ Pig } & avg (\%) & -0.78 & 0.09 & -1.12 \\
& best (\%) & 9.76 & 3.35 & 4.73 \\
\hline \multirow{2}{*}{ Galway } & avg (\%) & 1.31 & 0.76 & 0.62 \\
& best (\%) & 5.99 & 5.67 & 6.57 \\
\hline \multirow{2}{*}{ GOES } & avg (\%) & 4.37 & 3.01 & 3.57 \\
& best (\%) & 13.40 & 9.22 & 9.85 \\
\hline
\end{tabular}

TABLE IX

IMPROVEMENT IN SSIM USING APPROXIMATED RATE-DISTORTION BASED BIT ALLOCATION OVER EQUAL ALLOCATION. WAVELET PACKET COMPRESSION WAS USED TO COMPRESS RESIDUAL IMAGES.

\begin{tabular}{|c|l|r|r|r|}
\hline \multicolumn{2}{|c|}{} & Centroid & MST & MST $_{a}$ \\
\hline \multirow{2}{*}{ Joe } & avg (\%) & 0.29 & 0.21 & 0.17 \\
& best (\%) & 1.64 & 1.84 & 1.54 \\
\hline \multirow{2}{*}{ Pig } & avg (\%) & -1.81 & -0.22 & -0.49 \\
& best (\%) & 0.41 & 0.16 & 0.16 \\
\hline \multirow{2}{*}{ Galway } & avg (\%) & 0.84 & 0.34 & -0.04 \\
& best (\%) & 3.79 & 2.86 & 2.24 \\
\hline \multirow{2}{*}{ GOES } & avg (\%) & 2.79 & 0.65 & 0.64 \\
& best (\%) & 12.88 & 3.30 & 2.97 \\
\hline
\end{tabular}

The compression results are usually worse than those obtained when the rate-distortion curve is sampled at more bit rates, especially when the SSIM distortion mesaure and JPEG2000 is used. On the other hand, the approximated ratedistortion curve is considerably faster to compute. The general trend is the same whether the approximated rate-distortion curve is used-the improvement is higher at lower bit rates than at higher bit rates. When the RMSE and wavelet packet compression is used as a distortion measure, we see that the improvements are often comparable to those obtained without the approximated rate-distortion curve. This also agrees with previous works on image set compression that wavelet packet compression often performs better than JPEG2000 in image set compression [17].

The approximated rate-distortion curve can be obtained using a smaller number of invocations of the image compression and decompression algorithm, leading to a reduction of encoding time by approximately a factor of 4 . This improvement is particularly significant when wavelet packet compression is used, because the wavelet packet compression algorithm is more computationally intensive than JPEG2000. Thus, reducing the number of invocations to the compression algorithm is important.

\section{CONCLusions}

In this paper, we showed that a rate-distortion approach to optimize bit allocation can improve the performance of image set compression algorithms. While it is computationally intensive to obtain the rate-distortion curve for each residual image, we show that these curves can be approximated easily and still obtain a reasonable improvement in compression performance.

\section{ACKNOWLEDGMENT}

The authors wish to thank the Natural Sciences and Engineering Research Council of Canada for supporting this work through its Discovery Grant and Undergraduate Student Research Award programs.

\section{REFERENCES}

[1] S. Ait-Aoudia and A. Gabis, "A comparison of set redundancy compression techniques," EURASIP Journal on Applied Signal Processing, vol. 2006, pp. 1-13, 2006.

[2] C.-P. Chen, C.-S. Chen, K.-L. Chung, H.-I. Lu, and G. Tang, "Image set compression through minimal-cost prediction structures," in Proceedings of the IEEE International Conference on Image Processing, 2004, pp. 1289-1292.

[3] G. Jomier, M. Manouvrier, and M. Rukoz, "Storage and management of similar images." Journal of the Brazilian Computer Society, vol. 6, no. 3, pp. 13-25, 2000.

[4] K. Karadimitriou, "Set redundancy, the enhanced compression model, and methods for compressing sets of similar images," Ph.D. dissertation, Louisiana State University, 1996.

[5] K. Karadimitriou and J. M. Tyler, "Min-max compression methods for medical image databases." ACM Special Interest Group on Management of Data, vol. 26, no. 1, pp. 47-52, 1997.

[6] — "The centroid method for compressing sets of similar images," Pattern Recognition Letters, vol. 19, no. 7, pp. 585-593, 1998.

[7] Y. S. Musatenko and V. N. Kurashov, "Correlated image set compression system based on new fast efficient algorithm of Karhunen-Loeve transform," in Proceedings of The International Society for Optical Engineering: Multimedia Storage and Archiving Systems III, C.-C. J. Kuo, S.-F. Chang, and S. Panchanathan, Eds., October 1998, pp. 518529.

[8] C. Nielsen and X. Li, "MST for lossy compression on image sets," in Proceedings of the Data Compression Conference, 2006, p. 463.

[9] C. Nielsen, X. Li, and K. Abma, "Methods of grouping similar images for compression coding," in Proceedings of the International Conference on Computer Vision (WCAC'05), Las Vegas, June 20-23, 2005, pp. 93 99.

[10] V. Trivedi and H. Cheng, "Lossless compression of satellite image sets using spatial area overlap compensation," in Image Analysis and Recognition, 8th International Conference, ICIAR 2011, Part II, 2011, pp. 243-252.

[11] M. Vassilakopoulos and Y. Manolopoulos, "Dynamic inverted quadtree: a structure for pictorial databases," Information Systems, vol. 20, no. 6, pp. 483-500, 1995.

[12] M. Vassilakopoulos, Y. Manolopoulos, and N. Economou, "Overlapping quadtrees for the representation of similar images," Image and Vision Computing, vol. 11, no. 5, pp. 257-262, 1993.

[13] J. Wang and H. Yan, "Form image compression using template extraction and matching," in Proceedings of Visual Information Processing, December 2000.

[14] Z. Shi, X. Sun, and F. Wu, "Feature-based image set compression," in 2013 IEEE International Conference on Multimedia and Expo (ICME), July 2013, pp. 1-6.

[15] Y. Ling, O. Au, R. Zou, J. Pang, H. Yang, and A. Zheng, "Photo album compression by leveraging temporal-spatial correlations and HEVC," in 2014 IEEE International Symposium on Circuits and Systems (ISCAS), June 2014, pp. 1917-1920.

[16] A. Ortega and K. Ramchandran, "Rate-distortion methods for image and video compression," Signal Processing Magazine, IEEE, vol. 15, no. 6, pp. $23-50$, November 1998.

[17] B. Gergel, "Automatic compression for image sets using a graph theoretical framework," Master's thesis, University of Lethbridge, 2007.

[18] B. Gergel, H. Cheng, and X. Li, "A unified framework for lossless image set compression," in Data Compression Conference, 2006, p. 448.

[19] B. Gergel, H. Cheng, C. Nielsen, and X. Li, "A unified framework for image set compression," in Proceedings of the 2006 International Conference on Image Processing, Computer Vision, \& Pattern Recognition (IPCV'06), vol. II, 2006, pp. 417-423. 
[20] C. Lerner and H. Cheng, "Rate-distortion approach to bit allocation in lossy image set compression," in 2014 International Conference on Systems, Signals and Image Processing (IWSSIP), May 2014, pp. 227230.

[21] F. Meyer, A. Averbuch, and J. Strömberg, "Fast adaptive wavelet packet image compression," IEEE Transactions on Image Processing, vol. 9, no. 5, pp. 792-800, 2000.

[22] M. Adams, "JasPer project," http://www.ece.uvic.ca/ $/$ mdadams/jasper/.

[23] P. Merkle, K. Müller, A. Smolic, and T. Wiegand, "Efficient compression of multi-view video exploiting inter-view dependencies based on H.264/MPEG4-AVC," in IEEE Intl. Conf. on Multimedia and Expo (ICME'06), 2006, pp. 1717-1720.

[24] T. Frajka and K. Zeger, "Residual image coding for stereo image compression," Optical Engineering, vol. 42, no. 1, pp. 182-188, 2003.

[25] M. G. Perkins, "Data compression of stereopairs," IEEE Trans. on Communications, vol. 40, no. 4, pp. 684-696, 1992.

[26] R. C. Gonzalez and R. E. Woods, Digital Image Processing, 3rd ed. Prentice Hall, 2007.

[27] Z. Wang, A. Bovik, H. Sheikh, and E. Simoncelli, "Image quality assessment: From error visibility to structural similarity," IEEE Transactions on Image Processing, vol. 13, no. 4, pp. 600-612, 2004.

[28] D. Brunet, E. Vrscay, and Z. Wang, "A class of image metrics based on structural similarity quality index," in Image Analysis and Recognition (ICIAR) 2011, Part I, ser. Lecture Notes in Computer Science, no. 6753, 2011, pp. 100-110.

[29] H. Everett, "Generalized Lagrange multiplier method for solving problems of optimum allocation of resources," Operations Research, vol. 11, no. 3, pp. 399-417, 1963.

[30] S. Mallat and F. Falzon, "Analysis of low bit rate image transform coding," IEEE Transactions on Signal Processing, vol. 46, no. 4, pp. 1027-1042, Apr 1998.

[31] "JoeTourist Infosystems," http://www.JoeTourist.net.

[32] "Galway.net," http://www.galway.net/webcam/images.shtml.

[33] "GOES project science," http://rsd.gsfc.nasa.gov/goes. 